\title{
A canonical expansion of the product of two Stanley symmetric functions
}

\section{Nan Li}

Received: 1 September 2012 / Accepted: 9 August 2013 / Published online: 12 September 2013

(C) Springer Science+Business Media New York 2013

\begin{abstract}
We study the problem of expanding the product of two Stanley symmetric functions $F_{w} \cdot F_{u}$ into Stanley symmetric functions in some natural way. Our approach is to consider a Stanley symmetric function as a stabilized Schubert polynomial $F_{w}=\lim _{n \rightarrow \infty} \mathfrak{S}_{1^{n} \times w}$, and study the behavior of the expansion of $\mathfrak{S}_{1^{n} \times w} \cdot \mathfrak{S}_{1^{n} \times u}$ into Schubert polynomials as $n$ increases. We prove that this expansion stabilizes and thus we get a natural expansion for the product of two Stanley symmetric functions. In the case when one permutation is Grassmannian, we have a better understanding of this stability. We then study some other related stability properties, providing a second proof of the main result.
\end{abstract}

Keywords Schubert polynomials · Stanley symmetric functions · Maximal transition tree

\section{Introduction}

\subsection{Background and motivation}

In [21], Stanley defined a homogeneous power series $F_{w}$ in infinitely many variables $\left\{x_{1}, x_{2}, \ldots\right\}$ to compute the number of reduced decompositions of a given permutation $w$. He proved that $F_{w}$ is symmetric; $F_{w}$ is now referred to as a Stanley symmetric function. Here we use $F_{w}$ to denote $F_{w^{-1}}$ defined in [21]. It is shown in [7] that

$$
F_{w}=s_{D(w)},
$$

where $D(w)$ is the diagram of $w$ and $s_{D(w)}$ is the generalized Schur function defined in terms of the column-strict balanced labellings of $D(w)$. We are interested in the

N. Li (凶)

Department of Mathematics, Massachusetts Institute of Technology, Cambridge, MA 02139, USA

e-mail: nan@math.mit.edu 
problem of expanding the product of two Stanley symmetric functions $F_{w} \cdot F_{u}$ into Stanley symmetric functions. The hope is that we can explain the coefficients in terms of $D(w)$ and $D(u)$, as a generalized Littlewood-Richardson rule for Schur functions.

However, since the Stanley symmetric functions are not linearly independent, we want to expand them in some natural way. For $w \in S_{m}$ and $u \in S_{n}$, denote by $w \times u$ the permutation $v \in S_{m+n}$, with one line notation: $w(1) \cdots w(m)(u(1)+$ $m) \cdots(u(n)+m)$. Also, by $1^{n}$, we mean $1 \times 1 \times \cdots \times 1=123 \cdots n$. For example, $1^{2} \times 2134=124356$. We consider a Stanley symmetric function as a stabilized Schubert polynomial [16]:

$$
F_{w}=\lim _{n \rightarrow \infty} \mathfrak{S}_{1^{n} \times w} .
$$

Divided difference operators were first used by Bernstein-Gelfand-Gelfand [4] and Demazure [6] for the study of the cohomology of flag manifolds. Later, Lascoux and Shützenberger [12] developed the theory of Schubert polynomials based on divided difference operators. The collection $\left\{\mathfrak{S}_{w} \mid w \in S_{n}\right\}$ of Schubert polynomials corresponds to an integral basis for the cohomology ring of the flag manifold, and thus there exist integer structure constants $c_{w u}^{v}$ such that

$$
\mathfrak{S}_{w} \cdot \mathfrak{S}_{u}=\sum_{v} c_{w u}^{v} \mathfrak{S}_{v}
$$

It is a long standing question to find a combinatorial description of these constants. Some special cases are known. The simplest but important case is Monk's rule [18], which corresponds to the case when one of the Schubert polynomials is indexed by a simple transposition. A generalized Pieri rule was conjectured by Lascoux and Shützenberger [12], where they also sketched an algebraic proof. It was conjectured by Bergeron and Billey [3] in another form, and was proved by Sottile [20] using geometry, and by Winkel [22] via a combinatorial proof. There are also results about the case of a Schubert polynomial times a Schur polynomial, for example, see [10], [15], and [2].

In Sect. 2, we prove the main result Theorem 1.3 using the combinatorial definition of Schubert polynomials given in [5]. In Sect. 3, we study the case when one of the permutation is Grassmannian. We prove Theorems 1.3 and 1.4 by an algorithm described in [10] using maximal transitions (3.1).

For the case when both $w, u$ are Grassmannian, $\mathfrak{S}_{1^{n} \times w} \cdot \mathfrak{S}_{1^{n} \times u}$ is the product of two Schur polynomials for all $n$, so (1.2) is described by the usual LittlewoodRichardson rule. When both $w$ and $u$ are 321-avoiding, by [5], (1.2) gives an expansion of the product of two skew Schur functions. Compare this with the skew Littlewood-Richardson rule studied in [1] and [11], where they give a nice formula for the coefficients (with signs) in the expansion of two skew Schur functions into skew Schur functions. Here, we get all positive coefficients, but not all permutations appearing in the expansion are 321-avoiding. Therefore, our expansion is different from the one studied in [1] and [11].

In Sect. 4, we generalize this stability to the product of double Schubert polynomials. We also give the definition of the weak and strong stable expansions, and prove some other stable properties, which provide a second proof of Theorem 1.3. 


\subsection{Examples and results}

In order to study the expansion of $F_{w} \cdot F_{u}$, we study the behavior, as $n$ increases, of the expansion of $\mathfrak{S}_{1^{n} \times w} \cdot \mathfrak{S}_{1^{n} \times u}$ into Schubert polynomials. To motivate our main theorem, we will first give a canonical expansion of the product of a Stanley symmetric function $F_{w}$ with a Stanley symmetric function indexed by a transposition.

The following result is well-known.

Theorem 1.1 (Monk's rule [18])

$$
\mathfrak{S}_{w} \cdot \mathfrak{S}_{t_{m, m+1}}=\sum_{\substack{j \leq m<k \\ \ell\left(w t_{j k}\right)=\ell(w)+1}} \mathfrak{S}_{w t_{j k}}
$$

where $\ell(w)$ is the length of the permutation $w$ and $w t_{j k}$ is the permutation obtained from $w$ by exchanging $w(j)$ and $w(k)$.

Notice that $1 \times t_{m, m+1}=t_{m+1, m+2}$. Then for $\mathfrak{S}_{1 \times w} \cdot \mathfrak{S}_{1 \times t_{m, m+1}}$, we will have a term $\mathfrak{S}_{1 \times w t_{j k}}$ corresponding to each term $\mathfrak{S}_{w t_{j k}}$ in the expansion of $\mathfrak{S}_{w} \cdot \mathfrak{S}_{t_{m, m+1}}$. Let the position of 1 in $w$ be $s$, i.e., $w^{-1}(1)=s$. If $s \leq m$, then there are no more permutations; otherwise, if $s>m$, we get one more permutation $(1 \times w) t_{1, s+1}$. This holds for all $\mathfrak{S}_{1^{n} \times w} \cdot \mathfrak{S}_{1^{n} \times t_{m, m+1}}$. More precisely, we have

$$
\mathfrak{S}_{1^{n} \times w} \cdot \mathfrak{S}_{1^{n} \times t_{m, m+1}}=\sum_{\substack{j \leq m<k \\ \ell\left(w t_{j k}\right)=\ell(w)+1}} \mathfrak{S}_{1^{n} \times w t_{j k}}\left(+\mathfrak{S}_{1^{n-1} \times(1 \times w) t_{1, s+1}} \text {, if } s>m\right) .
$$

Now taking the limit for $n \rightarrow \infty$, we get the following canonical expansion:

$$
F_{w} \cdot F_{t_{m, m+1}}=\sum_{\substack{j \leq m<k \\ \ell\left(w t_{j k}\right)=\ell(w)+1}} F_{w t_{j k}}\left(+F_{(1 \times w) t_{1, s+1}} \text {, if } s>m\right) .
$$

Let us look at another example for $w=3241$ and $u=4312$.

Example 1.2 Consider $\mathfrak{S}_{1^{n} \times 3241} \cdot \mathfrak{S}_{1^{n} \times 4312}$ as $n$ increases. For $n=0,1,2$, we have

$$
\begin{aligned}
\mathfrak{S}_{3241} \cdot \mathfrak{S}_{4312}= & \mathfrak{S}_{642135} \\
\mathfrak{S}_{1 \times 3241} \cdot \mathfrak{S}_{1 \times 4312}= & \mathfrak{S}_{1 \times 642135} \\
& +\underline{\mathfrak{S}_{265314}+\mathfrak{S}_{2743156}+\mathfrak{S}_{356214}+\mathfrak{S}_{364215}+\mathfrak{S}_{365124}} \\
& \frac{+\mathfrak{S}_{462315}+\mathfrak{S}_{561324},}{\mathfrak{S}_{12 \times 3241} \cdot \mathfrak{S}_{1^{2} \times 4312}=} \\
& +\mathfrak{S}_{1^{2} \times 642135}+\mathfrak{S}_{1 \times 265314}+\mathfrak{S}_{1 \times 2743156}+\mathfrak{S}_{1 \times 356214} \\
& +\mathfrak{S}_{1 \times 364215}+\mathfrak{S}_{1 \times 365124} \\
& +\mathfrak{S}_{1 \times 462315}+\mathfrak{S}_{1 \times 561324}+\mathfrak{S}_{2375416}+\mathfrak{S}_{246531}+\mathfrak{S}_{256341} .
\end{aligned}
$$


As $n$ increases, the permutations appearing in the previous case reappear and add some new permutations (the underlined terms). In this example, the expansion stabilizes after $n=2$. One may check that

$$
\begin{aligned}
\mathfrak{S}_{1^{n} \times 3241} \cdot \mathfrak{S}_{1^{n} \times 4312}= & \mathfrak{S}_{1^{n} \times 642135}+\mathfrak{S}_{1^{n-1} \times 265314}+\mathfrak{S}_{1^{n-1} \times 2743156} \\
& +\mathfrak{S}_{1^{n-1} \times 356214}+\mathfrak{S}_{1^{n-1} \times 364215} \\
& +\mathfrak{S}_{1^{n-1} \times 365124}+\mathfrak{S}_{1^{n-1} \times 462315}+\mathfrak{S}_{1^{n-1} \times 561324} \\
& +\mathfrak{S}_{1^{n-2} \times 2375416}+\mathfrak{S}_{1^{n-2} \times 246531} \\
& +\mathfrak{S}_{1^{n-2} \times 256341} .
\end{aligned}
$$

Then taking $n \rightarrow \infty$, we have

$$
\begin{aligned}
F_{3241} \cdot F_{4312}= & F_{642135}+F_{265314}+F_{2743156}+F_{356214}+F_{364215} \\
& +F_{365124}+F_{462315}+F_{561324} \\
& +F_{2375416}+F_{246531}+F_{256341} .
\end{aligned}
$$

The stability of the expansion $\mathfrak{S}_{1^{n} \times w} \cdot \mathfrak{S}_{1^{n} \times u}$ we observed in the previous two examples is true in general. Here is the main result of this paper.

Theorem 1.3 Let $w$, u be two permutations.

1. Suppose $\mathfrak{S}_{w} \cdot \mathfrak{S}_{u}=\sum_{v_{0} \in V_{0}} c_{w, u}^{v_{0}} \mathfrak{S}_{v_{0}}$. Then

$$
\mathfrak{S}_{1 \times w} \cdot \mathfrak{S}_{1 \times u}=\sum_{v_{0} \in V_{0}} c_{w, u}^{v_{0}} \mathfrak{S}_{1 \times v_{0}}+\sum_{v_{1} \in V_{1}} c_{w, u}^{v_{1}} \mathfrak{S}_{v_{1}}
$$

where $v_{1}(1) \neq 1$, for each $v_{1} \in V_{1}$.

2. Let $k=\ell(w)+\ell(u)$. Then for all $n \geq k$, we have

$$
\begin{aligned}
\mathfrak{S}_{1^{n} \times w} \cdot \mathfrak{S}_{1^{n} \times u}= & \sum_{v_{0} \in V_{0}} c_{w, u}^{v_{0}} \mathfrak{S}_{1^{n} \times v_{0}}+\sum_{v_{1} \in V_{1}} c_{w, u}^{v_{1}} \mathfrak{S}_{1^{n-1} \times v_{1}}+\cdots \\
& +\sum_{v_{k} \in V_{k}} c_{w, u}^{v_{k}} \mathfrak{S}_{1^{n-k} \times v_{k}}
\end{aligned}
$$

where $V_{i}$ (possibly empty) is the set of new permutations appearing in $\mathfrak{S}_{1^{i} \times w}$. $\mathfrak{S}_{1^{i} \times u}$ compared to $\mathfrak{S}_{1^{i-1} \times w} \cdot \mathfrak{S}_{1^{i-1} \times u}$. Taking $n \rightarrow \infty$, we have a canonical expansion:

$$
F_{w} \cdot F_{u}=\sum_{v \in V} c_{w, u}^{v} F_{v}
$$

where $V=V_{0} \cup \cdots \cup V_{k}$.

For a permutation $w \in S_{n}$, define the code $c(w)$ to be the sequence $c(w)=$ $\left(c_{1}, c_{2}, \ldots\right)$ of nonnegative integers given by $c_{i}=\#\{j \in[n] \mid j>i, w(j)<w(i)\}$. 
Define the length of $c(w)$ to be $i_{0}=\max \left\{i \mid c_{i} \neq 0\right\}$, denoted by $\ell(c(w))$. We call a permutation Grassmannian if it has at most one descent. It is known that if $w$ is Grassmannian, then $\mathfrak{S}_{w}$ is a Schur polynomial in $\ell(c(w))$ variables.

Theorem 1.4 Using the notations from Theorem 1.3, if one of $w, u$ is Grassmannian, then:

1. If $V_{i}=\emptyset$ for some $i$, then $V_{j}=\emptyset$ for all $j>i$. We call the smallest $i$ such that $V_{i}=\emptyset$ the stability number for $w, u$.

2. The stability number is bounded by $\max \{\ell(c(w)), \ell(c(u))\}$. In particular, if $w=u$ with $w(1) \neq 1$, the stability number equals $w^{-1}(1)-1$.

Conjecture 1.5 Theorem 1.4 is true for general $w, u$.

\section{Proof of Theorem 1.3}

Let us recall the combinatorial definition of Schubert polynomials introduced in Theorem 1.1 [5]. Let $p=\ell(w)$ be the length of $w$, and $R(w)$ be the set of all the reduced words of $w$. For $a=\left(a_{1}, \ldots, a_{p}\right)$, let $K(a)$ be the set of all $a$-compatible sequences, i.e., $\left(i_{1}, \ldots, i_{p}\right)$ such that:

1. $i_{1} \leq \cdots \leq i_{p}$

2. $i_{j} \leq a_{j}$, for $j=1, \ldots, p$;

3. $i_{j}<i_{j+1}$, if $a_{j}<a_{j+1}$.

Then we have

$$
\mathfrak{S}_{w}=\sum_{a \in R(w)} \sum_{\left(i_{1}, \ldots, i_{p}\right) \in K(a)} x_{i_{1}} \cdots x_{i_{p}} .
$$

Definition 2.1 For two integer vectors $b^{1}=\left(b_{1}^{1}, \ldots, b_{p}^{1}\right)$ and $b^{2}=\left(b_{1}^{2}, \ldots, b_{p}^{2}\right)$, consider the following conditions:

1. $b^{1}$ and $b^{2}$ are weakly increasing. Namely, $b_{1}^{1} \leq \cdots \leq b_{p}^{1}$ and $b_{1}^{2} \leq \cdots \leq b_{p}^{2}$.

2. $b^{1}$ is smaller than $b^{2}$, denoted by $b^{1}<b^{2}$, which means $b_{i}^{1} \leq b_{i}^{2}$ for each $i=$ $1, \ldots, p$;

3. $b^{1}$ is similar to $b^{2}$, denoted by $b^{1} \sim b^{2}$, which means $b^{1}$ and $b^{2}$ increase at the same time, i.e., $b_{i}^{1}<b_{i+1}^{1}$ if and only if $b_{i}^{2}<b_{i+1}^{2}$; and

4. $b^{1}$ and $b^{2}$ are bounded by $n$, i.e., $b_{i}^{1} \leq n$ and $b_{i}^{2} \leq n$, for all $i=1, \ldots, p$.

We call $\left(b^{1}, b^{2}\right)$ a good pair if it satisfies the first three conditions and a good-n pair if all four conditions are satisfied.

For example, $\left(b^{1}, b^{2}\right)$, with $b^{1}=(2,4,4,5)$ and $b^{2}=(2,6,6,8)$, is a good-8 pair. Denote $X_{b}=x_{b_{1}} x_{b_{2}} \cdots x_{b_{p}}$. For example, $X_{b^{1}}=x_{2} x_{4}^{2} x_{5}$, for the previous $b^{1}$. We use $\operatorname{co}\left(X_{b}\right)$ to denote the coefficient of $X_{b}$. 


\section{Lemma 2.2}

1. In $\mathfrak{S}_{w}, \operatorname{co}\left(X_{b^{1}}\right) \geq \operatorname{co}\left(X_{b^{2}}\right)$, for any good pair $\left(b^{1}, b^{2}\right)$.

2. In $\mathfrak{S}_{1^{n} \times u}, \operatorname{co}\left(X_{b^{1}}\right)=\operatorname{co}\left(X_{b^{2}}\right)$, for any good-n pair $\left(b^{1}, b^{2}\right)$.

3. In $\mathfrak{S}_{1^{n} \times u}, \operatorname{co}\left(X_{b^{1}} \cdot g\right)=\operatorname{co}\left(X_{b^{2}} \cdot g\right)$, for any good-n pair $\left(b^{1}, b^{2}\right)$ and any monomial $g$ with variable indices larger than $n$. And

4. In $\mathfrak{S}_{1^{n} \times w} \cdot \mathfrak{S}_{1^{n} \times u}, \operatorname{co}\left(X_{b^{1}} \cdot g\right)=\operatorname{co}\left(X_{b^{2}} \cdot g\right)$, for any good-n pair $\left(b^{1}, b^{2}\right)$ any monomial $g$ with indices larger than $n$.

Proof Parts 1-3 follow from the combinatorial definition (2.1) of Schubert polynomials and Definition 2.1. Now we will prove part 4. In fact, any $X_{b^{1}} \cdot g$ is the product of two monomials, one from $\mathfrak{S}_{1^{n} \times w}$ and one from $\mathfrak{S}_{1^{n} \times u}$, let us assume $X_{b^{1}}=X_{b^{11}} \cdot X_{b^{12}}$, and the corresponding decomposition for $X_{b^{2}}$ is $X_{b^{2}}=X_{b^{21}} \cdot X_{b^{22}}$. Since $b^{1} \sim b^{2}$, we have $b^{11} \sim b^{21}$ and $b^{12} \sim b^{22}$. Applying part 3 to both pairs, we have $\operatorname{co}\left(X_{b^{1}} \cdot g\right)=\operatorname{co}\left(X_{b^{2}} \cdot g\right)$.

Example 2.3 For example, consider the previous good-8 pair $\left(b^{1}, b^{2}\right)$. If $X_{b^{1}}=$ $x_{2} x_{4}^{2} x_{5}=\left(x_{2} x_{4}\right)\left(x_{4} x_{5}\right)$ with $b^{11}=(2,4)$ and $b^{12}=(4,5)$, then we decompose $X_{b^{2}}=x_{2} x_{6}^{2} x_{8}$ as $\left(x_{2} x_{6}\right)\left(x_{6} x_{8}\right)$ with $b^{21}=(2,6)$ and $b^{22}=(6,8)$.

Write the code of $w$ as $c(w)=\left(c_{1}, c_{2}, \ldots, c_{p}\right)$ and $X^{c(w)}=x_{1}^{c_{1}} x_{2}^{c_{2}} \cdots x_{p}^{c_{p}}$. For any composition $c$, let $b(c)$ be the weakly increasing sequence such that $X_{b(c)}=X^{c}$. We use reverse lex-order in this section. It is known that the top degree term of $\mathfrak{S}_{w}$ is $X^{c(w)}$, i.e.,

$$
\mathfrak{S}_{w}=X^{c(w)}+\sum_{b} X_{b},
$$

where each $b$ satisfies $b<b(c(w))$, as defined in part 2 of Definition 2.1. Now we consider the process of getting the expansion of $\mathfrak{S}_{w} \cdot \mathfrak{S}_{u}$. By (2.1), the top degree term is $X^{c(w)+c(u)}$. Let $v_{1}$ be the permutations such that $c\left(v_{1}\right)=c(w)+c(u)$. Then

$$
\mathfrak{S}_{w} \cdot \mathfrak{S}_{u}=\mathfrak{S}_{v_{1}}+\cdots,
$$

so $c_{w u}^{v_{1}}=1$. Consider the top degree term in $\mathfrak{S}_{w} \cdot \mathfrak{S}_{u}-\mathfrak{S}_{v_{1}}$. Let it be $c_{2} X^{c\left(v_{2}\right)}$ for some $v_{2}$. Then

$$
\mathfrak{S}_{w} \cdot \mathfrak{S}_{u}-\mathfrak{S}_{v_{1}}=c_{2} \mathfrak{S}_{v_{2}}+\cdots
$$

Next, consider the top degree term in $\mathfrak{S}_{w} \cdot \mathfrak{S}_{u}-\mathfrak{S}_{v_{1}}-c_{2} \mathfrak{S}_{v_{2}}$, etc. Since there are finitely many monomials in $\mathfrak{S}_{w} \cdot \mathfrak{S}_{u}$, this process terminates, and we get an expansion $\mathfrak{S}_{w} \cdot \mathfrak{S}_{u}=\sum_{v \in V_{0}} c_{w u}^{v} \mathfrak{S}_{v}$.

Proof of Theorem 1.3 1. By the combinatorial definition of a Schubert polynomial (2.1) and the above process of expanding $\mathfrak{S}_{w} \cdot \mathfrak{S}_{u}$, we have $c_{1 \times w, 1 \times u}^{1 \times v}=c_{w, u}^{v}$ for all $v \in V_{0}$. Furthermore, each term in

$$
\mathfrak{S}_{1 \times w} \cdot \mathfrak{S}_{1 \times u}-\sum_{v_{0} \in V_{0}} c_{w, u}^{v_{0}} \mathfrak{S}_{1 \times v_{0}}
$$


is divisible by $x_{1}$. So any $\mathfrak{S}_{v}$ with $c(v)=\left(c_{1}, c_{2}, \ldots\right)$ appearing in the above difference has $c_{1} \neq 0$, which is equivalent to $v(1) \neq 0$. This proves part 1 .

2 . For a fixed $n$, suppose

$$
\mathfrak{S}_{1^{n} \times w} \cdot \mathfrak{S}_{1^{n} \times u}=\sum_{v \in V} c_{w u}^{v} \mathfrak{S}_{v}
$$

We claim that the code $c(v)=\left(c_{1}, c_{2}, \ldots, c_{p}\right)$ for $v \in V$ has to satisfy the following property: let $c(v)_{n}=\left(c_{1}, c_{2}, \ldots, c_{n}\right)$ be the first $n$ elements in $c(v)$. Let $i(v)$ be the smallest number such that $c_{i} \neq 0$. Then the claim is that if $i(v) \leq n$, then for all $i(v)<j \leq n$, we have $c_{j} \neq 0$. Suppose we have proved this claim. Then since $c_{1}+$ $\cdots+c_{n} \leq k$, where $k=\ell(w)+\ell(u)$, for each $v \in V$, we have $i(v)>n-k$. In other words, the code $c(v)$ starts with at least $n-k$ zeros, and thus $v$ starts with $12 \cdots(n-k)$, which will finish the proof. Now let us prove the claim.

In fact, suppose we have some $v_{0} \in V$ which does not satisfy the claim. Namely, there exists some $j$ such that $i(v)<j \leq n$ and $c_{j}=0$. Let $c^{\prime}=$ $\left(0, c_{1}, c_{2}, \ldots, c_{j-1}, c_{j+1}, \ldots, c_{n}\right)$. Consider the pair $b^{1}=b\left(c(v)_{n}\right)$ and $b^{2}=b\left(c^{\prime}\right)$, i.e., $X_{b^{1}}=X^{c(v)_{n}}$ and $X_{b^{2}}=X^{c^{\prime}}$. For example, let $n=7$, and $c\left(v_{0}\right)_{n}=(0,0,0,2,3$, $0,2)$. Then $X_{b^{1}}=X_{4}^{2} X_{5}^{3} x_{7}^{2}, c^{\prime}=(0,0,0,0,2,3,2)$ and $X_{b^{2}}=X_{5}^{2} X_{6}^{3} x_{7}^{2}$. Then $\left(b^{1}, b^{2}\right)$ is a good $n$-pair.

Now let $g=X^{\left(c_{n+1}, \ldots, c_{p}\right)}$. Notice that $X_{b^{1}} \cdot g$ is the top degree term in $\mathfrak{S}_{v_{0}}$ by (2.2). Since $b^{2}>b^{1}, \operatorname{co}\left(X_{b^{2}} \cdot g\right)=0$ in $\mathfrak{S}_{v_{0}}$. Therefore, $\operatorname{co}\left(X_{b^{1}} \cdot g\right)>\operatorname{co}\left(X_{b^{2}} \cdot g\right)$ in $\mathfrak{S}_{v_{0}}$. By Lemma 2.2, on the right hand side of the equation in Theorem 1.3, for each $v \in V$, we have $\operatorname{co}\left(X_{b^{1}} \cdot g\right) \geq \operatorname{co}\left(X_{b^{2}} \cdot g\right)$. Therefore, on the right hand side, we have $\operatorname{co}\left(X_{b^{1}} \cdot g\right)>\operatorname{co}\left(X_{b^{2}} \cdot g\right)$. However, on the left hand side, we must have $\operatorname{co}\left(X_{b^{1}} \cdot g\right)=\operatorname{co}\left(X_{b^{2}} \cdot g\right)$, a contradiction.

\section{Schubert polynomial times a Schur polynomial}

In this section, we will prove Theorems 1.3 and 1.4 for the case when one of the permutations $w, u$ is Grassmannian. We will apply an algorithm for multiplying a Schubert polynomial by a Schur polynomial based on the following result. This result was originally proved using Kohnert's algorithm. So this section is based on the following theorem. ${ }^{1}$

Theorem 3.1 (Theorem 3.1 in [10]) Let $\mathfrak{S}_{u}$ be a Schur polynomial with $m$ variables, i.e., $u$ is a Grassmannian permutation with $\ell(c(u))=m$. Let $\mathfrak{S}_{w}$ be a Schubert polynomial with $m$ variables, i.e., $\ell(c(w))=m$. Then

$$
\mathfrak{S}_{w} \cdot \mathfrak{S}_{u}=\mathfrak{S}_{w \times u} \downarrow A_{m},
$$

where $f \downarrow A_{m}=f\left(x_{1}, \ldots, x_{m}, 0, \ldots, 0\right)$.

\footnotetext{
${ }^{1}$ We have heard from a number of experts that there is some question about the validity of the proof of Theorem 3.1 in [10].
} 
The algorithm we will apply for multiplying a Schubert polynomial by a Schur polynomial was studied in [10] and is a modification of the algorithm by Lascoux and Schützenberger [13] for decomposing the product of two Schur functions into a sum of Schur functions.

\subsection{Maximal transition tree}

Recall that $w t_{r s}$ is the permutation obtained from $w$ by switching $w(r)$ and $w(s)$. Let $r$ be the largest descent of the permutation $w$, and $s$ be the largest integer such that $w(s)<w(r)$. The following formula follows from Monk's rule [18]

$$
\mathfrak{S}_{w}=x_{r} \mathfrak{S}_{u}+\sum_{v \in S(w)} \mathfrak{S}_{v},
$$

where $u=w t_{r s}$ and $S(w)$ is the set of permutations of the form $w t_{r s} t_{j r}$ with $j<r$ such that $\ell\left(w t_{r s} t_{j r}\right)=\ell(w)$. Denote the set of all possible $j$ 's to be $J(w)$. So each $v \in S(w)$ corresponds to a different $j \in J(w)$. We call (3.1) a maximal transition (MT for short) (see [13]). We call each $v \in S(w)$ a descendent of $w$.

Example 3.2 For example, for $w=321654$, we have $r(w)=5, s(w)=6, J(w)=$ $\{1,2,3\}$ and $S(w)=\{421635,341625,324615\}$.

Notice that $c_{i}=0$, for all $i>r(w)$ in the code $c(w)=\left(c_{1}, c_{2}, \ldots\right)$, and $\mathfrak{S}_{w}$ is a polynomial with $r(w)$ variables. So if $r(w) \leq m$, then $\mathfrak{S}_{w}=\mathfrak{S}_{w} \downarrow A_{m}$. If $r(w)>m$, we have $\mathfrak{S}_{w} \downarrow A_{m}=\sum_{v \in S(w)} \mathfrak{S}_{v} \downarrow A_{m}$ by (3.1), since we set $x_{r}=0$. Notice that for each permutation $v \in S(w), r(v)<r(w)$. We call a permutation $v$ bad if $v^{-1}(1)>$ $m+1$. If $v$ is bad, then $x_{m+1}$ divides each monomial of $\mathfrak{S}_{v}$, so $\mathfrak{S}_{v} \downarrow A_{m}=0$.

Apply MT successively to $w \times u$, each $v \in S(w \times u)$ and their descendants as long as the permutation is not bad, until their largest descents are smaller than $m$. This way we get a finite tree with two types of leaves: (i) a permutation with largest descent $\leq m$, we call it a good leaf; and (ii) a bad permutation as defined above. Then $\mathfrak{S}_{w \times u} \downarrow A_{m}$ is obtained by summing up all of the good leaves. We call this tree the MT-tree rooted at $w \times u$; we call the edge between a permutation $w$ and one of its descendant $v \in S(w)$ an MT-move.

Example 3.3 Here is an example of the MT tree rooted at $w \times u$, for $w=321$, $u=2413$ and $m=2$ (see Fig. 1). The leaves we cross out are the bad leaves, i.e., permutations with 1 in position larger than $m+1=3$. The remaining leaves are good leaves, i.e., they have largest descent $\leq m=2$. So summing up all the good leaves, we have $\mathfrak{S}_{321} \cdot \mathfrak{S}_{2413}=\mathfrak{S}_{321 \times 2413} \downarrow A_{2}=\mathfrak{S}_{53124}+\mathfrak{S}_{45123}$.

Fig. 1 MT-tree rooted at $321 \times 2413$ for Example 3.3

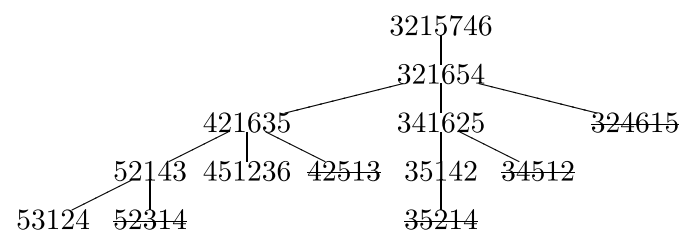


Fig. 2 MT-tree rooted at $1 \times 321 \times 1 \times 2413$ for Example 3.5

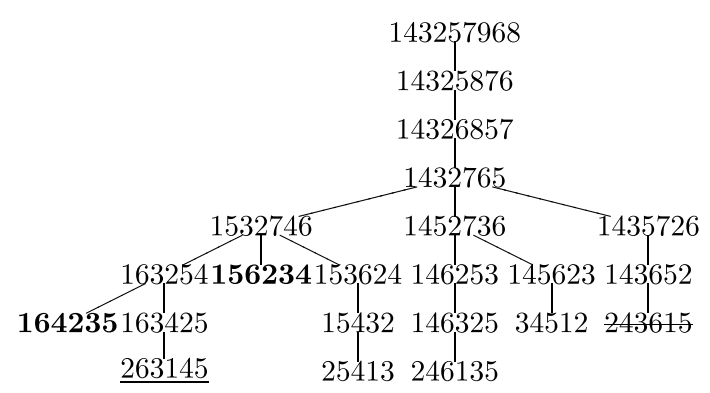

Remark 3.4 Notice that in Fig. 1, the descendants of 341625 are bad leaves (35214 and 34512 ). It will be nice if one could simplify the tree so that we can remove 341625 without applying further moves.

Now we want to study the difference between the MT-tree rooted at $1 \times w \times 1 \times u$ and the one rooted at $w \times u$.

Example 3.5 Continuing Example 3.3. We study $\mathfrak{S}_{1 \times 321} \cdot \mathfrak{S}_{1 \times 2413}$ (see Fig. 2). Notice that now $m=3$ instead of 2 in Example 3.3. Summing up all good leaves, we have $\mathfrak{S}_{1 \times 321} \cdot \mathfrak{S}_{1 \times 2413}=\mathfrak{S}_{1432 \times 13524} \downarrow A_{3}=\mathfrak{S}_{164235}+\mathfrak{S}_{156234}+\mathfrak{S}_{263145}+$ $\mathfrak{S}_{25413}+\mathfrak{S}_{246135}+\mathfrak{S}_{34512}$.

Compare the leaves of the above tree and those in Example 3.3. We have the following observations.

1. The good leaves in Example 3.3 (53124 and 45123) stay good in Example 3.5, simply with a one added in front $(1 \times 53124=164235$ and $1 \times 45123=156234$, bold in Fig. 2).

2. The remaining good leaves in Example 3.5 are descendants of some bad leaves in Example 3.3. For example, 263145 (underlined in Fig. 2) is obtained from 52314 which used to be bad in Example 3.3.

3. For the new good leaves in Example 3.5, the position of 1 stays the same as their ancestor in Example 3.3. For example, both 263145 and 52314 have 1 in the fourth position.

In general, the first and second observations above are true as a consequence of Lemma 3.6 (we will prove it in the next subsection), and the third observation is true by Lemma 3.7 .

Lemma 3.6 For the same $m$ as for $w$, $u$, the leaves ("good" and "bad") of $w \times 1 \times u$ are the same as leaves of $w \times u$.

Lemma 3.7 For any reduced permutation $w$ (cannot make more MT-moves), if we add 1 in the beginning and then apply the MT-moves to $1 \times w$, the position of 1 in the leaves is the same as the position of 1 in $w$.

Proof Let $r_{0}$ be the last descent of $w, s_{0}$ be the largest number such that $w\left(s_{0}\right)<$ $w\left(r_{0}\right) . w$ is reduced implies that in $w, J(w)=\emptyset$. Then since $r_{0}$ is the last descent, 
we can see that $w\left(r_{0}+1\right)=1$. Then in the first move for $1 \times w$, we will have $j=1$, $r=r_{0}+1$ thus and move 1 to the position of $r_{0}+1$. After this move, all the rest will not change the position of 1 . So 1 will be in the position $r_{0}+1$, which is the same as the position of 1 in $w$. After this move, all the rest will not change the position of 1 .

Now notice that in Example 3.5, there is still one bad leaf 243615 (see Fig. 2). So in the next step $\mathfrak{S}_{1^{2} \times 321} \cdot \mathfrak{S}_{1^{2} \times 2413}$, there will be some more good leaves with 243615 as ancestor. After that, the expansion $\mathfrak{S}_{1^{n} \times 321} \cdot \mathfrak{S}_{1^{n} \times 2413}$, for $n \geq 2$ should have no more new permutations. And in fact, this is the case: $\mathfrak{S}_{1^{n} \times 321}$. $\mathfrak{S}_{1^{n} \times 2413}=\mathfrak{S}_{1^{n} \times 53124}+\mathfrak{S}_{1^{n} \times 45123}+\mathfrak{S}_{1^{n-1} \times 263145}+\mathfrak{S}_{1^{n-1} \times 25413}+\mathfrak{S}_{1^{n-1} \times 246135}+$ $\mathfrak{S}_{1^{n-1} \times 34512}+\mathfrak{S}_{1^{n-2} \times 236415}$, for all $n \geq 2$. So we have

$$
F_{321} \cdot F_{2413}=F_{53124}+F_{45123}+F_{263145}+F_{25413}+F_{246135}+F_{34512}+F_{236415} \text {. }
$$

So the stability number for $\mathfrak{S}_{321} \cdot \mathfrak{S}_{2413}$ is 2 , as predicted by Theorem 1.4 part 2 that it should be bounded by $\ell(c(321))=\ell(c(2413))=2$. Now look at the positions of 1 in each permutation appearing on the right hand side of (3.2): $I=\{3,4,5\}$, which is an interval without any gaps. In general, we have

Lemma 3.8 Let $F_{w} \cdot F_{u}=\sum_{v \in V} F_{v}$ be the expansion we get by Theorem 1.3. Let $I\left\{v^{-1}(1) \mid v \in V\right\}$. Then $I=[a, b]$ an interval without any gaps.

Lemma 3.8 together with Theorem 1.3 will imply Theorem 1.4 . For a proof of Lemma 3.8, we also want to use the diagrams interpretation of the MT-move studied in the next subsection.

Lemma 3.9 If a permutation is reduced, then there are no descents after 1 . In other words, the length of the code is the number of boxes in the first column.

Proof Suppose there is a descent after 1, then it is not hard to see that this permutation is not reduced, since there must exist a $j$ for which we can apply MT-move.

Now assume that $\mathfrak{S}_{u}$ is a Schur polynomial and $\mathfrak{S}_{w}$ is a Schubert polynomial both in $m$ variables. Use the MT algorithm, we can show the result in both Theorems 1.3 and 1.4.

Proof of Theorem 1.3 1. Consider the expansion of $\mathfrak{S}_{1 \times w} \times \mathfrak{S}_{1 \times u}$ by looking at the tree rooted at $1 \times w \times 1 \times u$. By definition, all the good leaves for the tree rooted at $v \times u$ satisfy: (i) the last descents are no larger than $m$, and (ii) positions of the letter 1 are not larger than $m+1$. By Lemma 3.6, the leaves of $v \times 1 \times u$ are the same as the leaves for $v \times u$, both the good leaves and the bad leaves. So it is not hard to see that the leaves of $1 \times v \times 1 \times u$ are just the leaves of $v \times 1 \times u$ with an 1 added to the front. Now for $n=1+\ell$, the good leaves of $v \times u$ are still the good leaves for $1 \times v \times 1 \times u$, just with an 1 added to the front. Now for those bad leaves, by Lemma 3.7, after we add 1 to the front, and continue to apply the MT-moves, the position of 1 will not change. But because the number of the variables in $\mathfrak{S}_{1 \times w}$ and $\mathfrak{S}_{1 \times u}$ is now 
$m+1$ instead of $m$, some of the bad leaves (when the position of 1 is $m+2$ ) will become good leaves. Moreover, these newly added good leaves will not start with 1 . So the Schubert polynomials appeared in the expansion of $X_{1 \times v} \times X_{1 \times u}$ are indexed by the old good leaves, which all start with 1 , and possibly some new good leaves, which all do not start with 1 . This proves the first part.

2. When we append more ones in front of $u$ and $v$, all leaves of the tree will become good leaves, but there are only finitely many of them. So finally the expansion will be stable.

Proof of Theorem 1.4 1. Consider the diagram of a permutation (see the next subsection for details). By MT algorithm, if we are able to move a portion with length $s_{1}$ and $s_{2}$ to the first column, then we are able to move a portion with any length between $s_{1}$ and $s_{2}$. So there is no gap when new terms showing up in the expansion when $n$ increases. Applying different choices of $j$ gives us different number of potential boxes to be added to the first column. But this number should have no gap: if there are cases when there are one boxes left and four boxes left. Then there must be some combination of choices of $j$ 's such that there are two and three boxes left to be added to the first column.

2. By Lemma 3.9, for all leaves, the length of the code is the length of the first column. It is clear that the longest possible first column is the sum of the length of $c(w)$ and $c(u)$. From the diagrams perspective of the MT-moves developed in the next subsection, it is not hard to see that (refer to Fig. 4 for visualization), the stable number bounded by the maximal length of the first column minus $c(w)$. Therefore, we get a bound by the maximal length of $c(w)$ and $c(u)$. In the case $w=u$, and both being Grassmannian, we have $m=w^{-1}(1)-1$, which is exactly the stable number.

\subsection{MT-move in terms of diagrams}

In order to prove Lemmas 3.6 and 3.8, we want to describe the MT-move in terms of diagrams.

First, there is a correspondence between the set of inversions of $w$ and the boxes in the diagram. An inversion in a permutation $w$ is a pair of $(i, j)$ such that $i<j$ and $w^{-1}(i)>w^{-1}(j)$. We denote a box of the diagram in the $i$ th row and $j$ th column by $B_{i j}$. Then the box $B_{i j}$ corresponds to the inversion $(j, w(i))$ in $w$. For example, here is the diagram for $w=3215746$ (see Fig. 3(a)). The box $B_{56}$ (indicated by a bullet) corresponds to the inversion $(6,7)$ in $w$.

Now we study the MT-move in terms of diagrams. Let $v$ be a descendant of $w$ via an MT-move. Then $D(v)$ is obtained from $D(w)$ by moving some part of the diagram

Fig. 3 MT-move

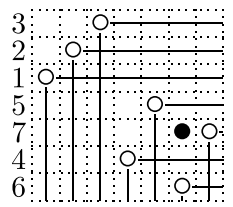

(a) $\mathrm{w}$

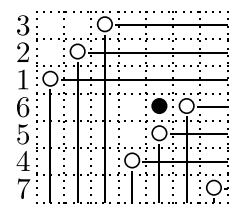

(b) $\mathrm{v}$ 


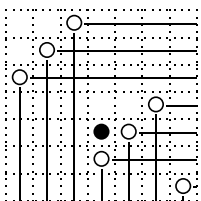

(a) $D(w)$

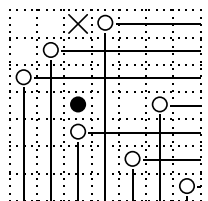

(b) $v_{1}(j=1)$

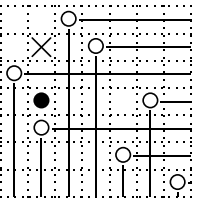

(c) $v_{2}(j=2)$

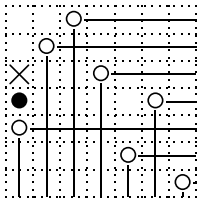

(d) $v_{3}(j=3)$

Fig. 4 MT-moves using different $j$ 's

up and left. For example, as shown in the first step of Example 3.3, applying an MTmove to $w=3215746$, we get $v=3216547$, and the diagram of $v$ is obtained from $D(w)$ by moving the box with a bullet up and left by one row and one column (see Fig. 3). Notice that this diagram move is very similar to the move described in [9].

Recall that $v=w t_{r s} t_{s j}$, where $r$ is the largest descent of $w, s$ is the largest number $s>r$ such that $w(s)<w(r)$, and $j$ is some number $j<r$ such that $w t_{r s} t_{s j}$ has the same length as $w$. From $w$ to $v=w t_{r s} t_{s j}$, we have the following change of inversions:

1. Change each inversion $(w(i), w(s))$ to an inversion $(w(i), w(j))$, for $j<i \leq r$. In terms of diagrams, this corresponds to moving the part of column $w(s)$ left to column $w(j)$, where the part is from the $(j+1)$ th row to the $r$ th row.

2. Change each inversion $(w(r), w(i))$ to $(w(j), w(i))$, for $r<i<s$ and $w(i)>$ $w(j)$. In terms of diagrams, this corresponds to moving the part of row $r$ with column indices in $\{w(i) \mid w(i)>w(j), r<i<s\}$ up to row $j$.

In the diagram of $w$, consider the right-down corner box $B(w)$, i.e., the box in the rightmost column of the lowest row. By the definition of $r(w)=r$ and $s(w)=s$, we have $B(w)=B_{r, w(s)}$. For each $j \in J(w)$, denote the box $B_{j, w(j)}$ by $T(w, j)$. Then the above changes of inversions can be seen as moving some blocks with $B(w)$ as its right-down corner up and left so that $T(w, j)$ becomes its upper-left corner. For example, consider $w=321654$ in the branching part of Example 3.3, with $J(w)=\{1,2,3\}$. See Fig. 4(a) for $D(w)$, where $B(w)$ is marked with a bullet and all three possible $T(w, j)$ 's are marked with $\times$. Now applying MT-moves to $D(w)$, all three $D(v)$, for $v \in S(w)$ are shown in Figs. 4(b), 4(c), and 4(d). Using this diagram interpretation of the MT-move, we can prove Lemma 3.6 by comparing the MT-moves of $D(w \times u)$ and $D(1 \times w \times 1 \times u)$.

Proof of Lemma 3.6 Compare the diagrams of $w \times u$ and $w \times 1 \times u$ (see Example $3.10)$. The two diagrams are basically the same, but because of the " 1 " in the middle of $w \times 1 \times u$, the boxes corresponding to $u$ are down by one row and right by one column. We call them delayed boxes. Now we apply the maximal transitions to $t:=$ $w \times 1 \times u$ and compare the moves to those for $w \times u$. As in the previous discussion, we start from the rightmost box $B$ (marked with a bullet) in the lowest row, which is in row $r$ and column $w(t)$. We will move some part of the diagram with $B$ as its right-down corner of the diagram up and left so that the box $T$ in row $j$ and column $w(t)$ becomes its up-left corner. Compare each move of $s$ to the moves of $w \times u$. There are two cases: 
1. The corresponding box for $B$ in $w \times u$ is also the rightmost box in the lowest row. In Example 3.10, $A_{i}$ and the last $B_{i j}$ in its row has the same $B$ box ( $A_{1}$ and $B_{12}$, $A_{2}$ and $B_{22}$, etc.).

2. In the lowest row, some delayed boxes are to the right of the box $B$ for $w \times u$. In Example 3.10, every $B_{i j}$ not belonging to the previous case is in this case.

Therefore, as we apply maximal transition to $w \times 1 \times u$, if the delayed boxes are not on the way, we can apply the same move as for $w \times u$; if we are not so lucky, we need to clear our way by moving all the delayed boxes up and left first. There are two important things to notice for this case: (i) there is only one possible $j$ to use (and always $j>m$ ), and it is exactly one row up and one column left to the delayed block that we need to move; (ii) after moving this delayed block up and left by one, the boxes in this block are no longer delayed. In other words, this cleaning work will not affect the actually moving work, and this cleaning work is finite. Once we finish all the cleaning work, we will get the exactly the same permutation in the process of $w \times u$, as in the example $A_{5}=B_{52}$.

Now it is left to show that all the cleaning work can be done before we get to the leaves of $w \times u$. Consider the condition when we get to a leaf: (i) all boxes are above the $(m+1)$ th row (good leaves) or (ii) the first column has more than $m$ boxes (bad leaves). If there are still some delayed boxes, since in the process of cleaning $j>m$, it is not possible that all boxes are above the $(m+1)$ th row. In the second case, assume that the first column already has more than $m$ boxes, but we still have delayed boxes. Since these delayed boxes are not in the first column, we can still do the cleaning until there are no delayed boxes left.

Example 3.10 Here is an example for $w=1^{2} \times 32154$ and $u=698435127$ with $m=6$. We start from $A_{1}=w \times u$ and $B_{11}=w \times 1 \times u$. Here are their diagrams (for simplicity, we ignore the $1^{2}$ in the front.)
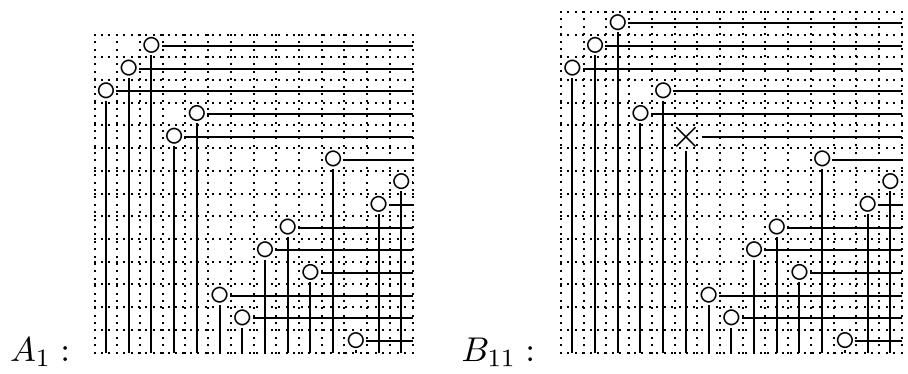

Then we apply MT-moves to both of them. Here is part of the tree. 


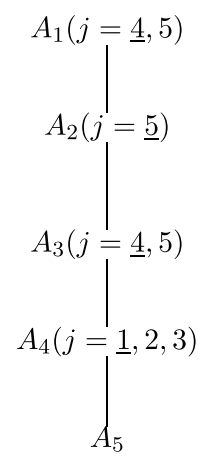

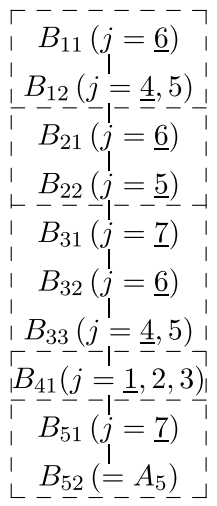

Even though the path of the $B_{i}$ 's is longer, eventually it gets to the same permutation $B_{52}=A_{5}$. For example, consider $A_{3}$ and $B_{31}$. In $A_{3}$, the corner box is indicated by a bullet. While in $B_{31}$, the corresponding box is also indicated by a bullet. But it is not the corner box in $B_{31}$ since there are some delayed boxes to its right (indicated by the dots). So we need to do some cleaning (the move from $B_{31}$ to $B_{32}$, and then to $B_{33}$ ) before moving the same block as $A_{3}$. Finally, in $B_{33}$, we are ready to move the same block as $A_{3}$.

$A_{3}$ :

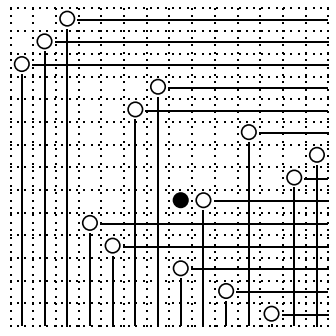

$B_{32}$ :

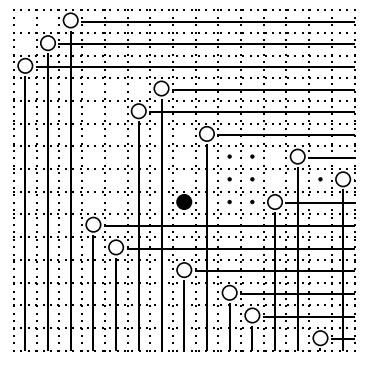

$B_{31}$ :
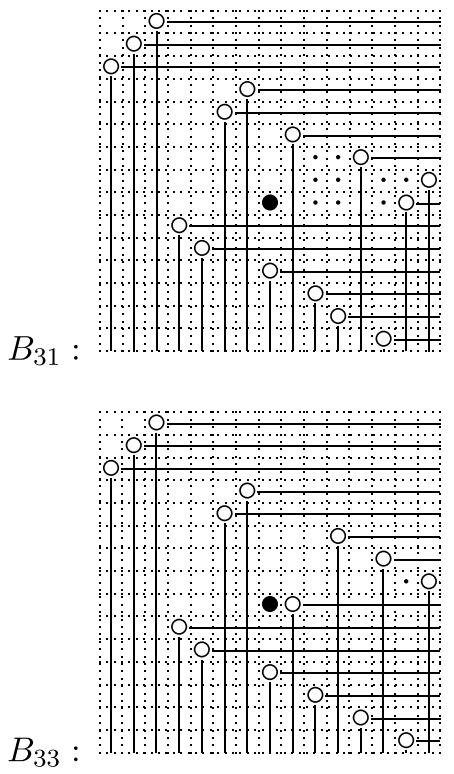

Proof of Lemma 3.8 Notice that $v^{-1}(1)-1$ is the number of boxes in the first column of $D(v)$. Consider again $w$ and $S(w)$ shown in Fig. 4. Notice that applying different $j \in J(w)$ may result in different numbers $b(j)$ of potential boxes to be added to the 
first column. For example, for $j=2$, there is one box left, and for $j=3$, there are two boxes left (and are already added). The set $b=\{b(j) \mid j \in J(w)\}=[1,2]$ is an interval without any gaps. Using the diagram interpretation of the MT-move, we can show that this holds in general, which implies this lemma. In fact, by the definition of $J(w)$, each $j \in J(w)$ has the following property: there is no element $w(i)$ with $j<i<r$ such that $w(j)<w(i)<w(r)$. In terms of the diagrams, this means that all boxes in the block between $w(j)$ and $w(r)$, i.e., the block with $w(j)$ as the upperleft corner and $w(r)$ as the lower-right corner, are crossed out. Therefore, suppose $j_{1}, j_{2}$ are the $k$ th and $(k+1)$ th largest in $J(w)$, then $b\left(j_{1}\right)=b\left(j_{2}\right)-1$. Therefore, $b=\{b(j) \mid j \in J(w)\}$ is an interval without any gaps, thus completing the proof of this lemma.

Corollary 3.11 Let $w, u$ be two permutations both with $\ell(c(w))=\ell(c(u))=m$ (for the case when $\ell(c(w)) \neq \ell(c(u))$, add enough ones to the front of one permutation). Assume $u$ is Grassmannian. Apply MT-moves successively to $D\left(1^{m} \times w \times u\right)$. Stop applying MT-moves to a diagram $D$ as soon as all the boxes in its diagram are in the first $2 m$ rows. Denote the multiset of the diagrams obtained this way by A. Then in the canonical expansion (1.2) $F_{w} \cdot F_{u}=\sum_{v \in V} c_{w u}^{v} F_{v}$, we have

$$
c_{w u}^{v}=\#\{D \in A \mid D=D(v)\} .
$$

\section{Other stable expansions}

In this section, we study some other stable expansions related with Schubert polynomials. Given a unique expansion, we study the behavior of that expansion when we embed $w \mapsto 1^{n} \times w$, as we did for Theorems 1.3 and 1.4. We call the eventually stabilized behavior as described in Theorem 1.3 weak stable property; and if it further satisfies the property that once there are no new terms, there will be no new terms ever, as described in Theorem 1.4, we call it strong stable property. First, as a direct corollary of Theorem 1.3, we have

Corollary 4.1 For the unique expansion of the product of finitely many Schubert polynomials into Schubert polynomials, we have the weak stable property, i.e.,

1. Suppose $\mathfrak{S}_{w_{1}} \cdots \mathfrak{S}_{w_{\ell}}=\sum_{v_{0} \in V_{0}} c_{w_{1}, \ldots, w_{\ell}}^{v_{0}} \mathfrak{S}_{v_{0}}$. Then

$$
\mathfrak{S}_{1 \times w_{1}} \cdots \mathfrak{S}_{1 \times w_{\ell}}=\sum_{v_{0} \in V_{0}} c_{w_{1}, \ldots, w_{\ell}}^{v_{0}} \mathfrak{S}_{1 \times v_{0}}+\sum_{v_{1} \in V_{1}} c_{w_{1}, \ldots, w_{\ell}}^{v_{1}} \mathfrak{S}_{v_{1}},
$$

where $v_{1}(1) \neq 1$, for each $v_{1} \in V_{1}$.

2. Let $k=\ell\left(w_{1}\right)+\cdots+\ell\left(w_{\ell}\right)$. Then for all $n \geq k$, we have

$$
\begin{aligned}
\mathfrak{S}_{1^{n} \times w_{1}} \cdots \mathfrak{S}_{1^{n} \times w_{\ell}}= & \sum_{v_{0} \in V_{0}} c_{w_{1}, \ldots, w_{\ell}}^{v_{0}} \mathfrak{S}_{1^{n} \times v_{0}}+\sum_{v_{1} \in V_{1}} c_{w_{1}, \ldots, w_{\ell}}^{v_{1}} \mathfrak{S}_{1^{n-1} \times v_{1}}+\cdots \\
& +\sum_{v_{k} \in V_{k}} c_{w_{1}, \ldots, w_{\ell}}^{v_{k}} \mathfrak{S}_{1^{n-k} \times v_{k}},
\end{aligned}
$$


where $V_{i}$ (possibly empty) is the set of new permutations appearing in $\mathfrak{S}_{1^{i} \times w_{1}} \ldots$ $\mathfrak{S}_{1^{i} \times w_{\ell}}$ compared to $\mathfrak{S}_{1^{i-1} \times w} \cdot \mathfrak{S}_{1^{i-1} \times u}$.

\subsection{Product of double Schubert polynomials}

For the double Schubert polynomials, we have the following connection to Schubert polynomials (for example, see Proposition 2.4.7 in [17])

$$
\mathfrak{S}_{w}(x, y)=\sum_{\begin{array}{c}
w=v^{-1} u \\
\ell(w)=\ell(u)+\ell(v)
\end{array}} \mathfrak{S}_{u}(x) \mathfrak{S}_{v}(-y) .
$$

Then consider the product of two double Schubert polynomials

$$
\begin{gathered}
\mathfrak{S}_{1^{n} \times w}(x, y) \mathfrak{S}_{1^{n} \times w^{\prime}}(x, y)=\sum_{\begin{array}{c}
w=v^{-1} u \\
\ell(w)=\ell(u)+\ell(v) \\
w^{\prime}=v^{\prime-1} u^{\prime} \\
\ell\left(w^{\prime}\right)=\ell\left(u^{\prime}\right)+\ell\left(v^{\prime}\right) \\
\times \mathfrak{S}_{1^{n} \times v^{\prime}}(-y) .
\end{array}} \mathfrak{S}_{1^{n} \times u}(x) \mathfrak{S}_{1^{n} \times v}(-y) \mathfrak{S}_{1^{n} \times u^{\prime}}(x) \\
\end{gathered}
$$

By Corollary 4.1, we have

Corollary 4.2 For the unique expansion of the product of finitely many double Schubert polynomials into Schubert polynomials, we have the weak stable property.

4.2 Stable expansion between $\mathfrak{S}_{w}$ and $e_{I}$

We write (2.1) as

$$
\mathfrak{S}_{w}=\sum_{a \in \mathbf{N}^{\infty}} K_{w, a} X^{a}
$$

where each $K_{w, a}$ is a nonnegative integer, and $K=\left(K_{w, a}\right)$ is known as the SchubertKostka matrix. Let

$$
e_{i}^{k}=\sum_{1 \leq r_{1}<\cdots<r_{i} \leq k} x_{r_{1}} \cdots x_{r_{i}},
$$

and for $I=\left(i_{1}, i_{2}, \ldots, i_{n}\right)$, let

$$
e_{I}=e_{i_{1}}^{1} e_{i_{2}}^{2} \cdots e_{i_{n}}^{n}
$$

Notice that $e_{i}^{k}=0$, if $i>k$, so we require $i_{k} \leq k$ in $I$.

The following result is well known.

Proposition 4.3 (See [12], [14, (2.6)-(2.7)], [8, (4.13)]) We have the following Zlinear bases for $\mathbf{Z}\left[x_{1}, \ldots, x_{n}\right] / I_{n}$, and each of them spans the same vector space which is complementary to $I_{n}$ :

1. The monomials $x_{1}^{a_{1}} \cdots x_{n-1}^{a_{n-1}}$ such that $0 \leq a_{k} \leq n-k$; 
2. The standard elementary monomials $e_{i_{1} i_{2} \cdots i_{n-1}}$;

3. The Schubert polynomials $\mathfrak{S}_{w}$ for $w \in S_{n}$.

By Proposition 4.3, we have the unique expansions of $e_{I}$ into $\mathfrak{S}_{w}$ and $\mathfrak{S}_{w}$ into $e_{I}$. In this subsection, we will prove the stable property for these two unique expansions.

Proposition 4.4 For $I=\left(i_{1}, \ldots, i_{n}\right)$, we have the strong stable property for the expansion

$$
e_{I}=\sum_{w \in W} \beta_{w}^{I} \mathfrak{S}_{w}
$$

i.e., for all $k \geq r=i_{1}+i_{2}+\cdots+i_{n}-n$, we have

$$
e_{\left(0^{k}, i_{1}, i_{2}, \ldots, i_{n}\right)}=\sum_{w \in W} \beta_{w}^{I} \mathfrak{S}_{1^{k} \times w}+\sum_{w_{1} \in W_{1}} \beta_{w_{1}}^{I} \mathfrak{S}_{1^{k-1} \times w}+\cdots+\sum_{w_{k} \in W_{r}} \beta_{w_{r}}^{I} \mathfrak{S}_{1^{k-r} \times w},
$$

where for $i=1, \ldots, r$, we have $W_{i} \neq \emptyset$ and $W_{r}$ is the single permutation $w_{r}=$ $23 \cdots(r+n+1) 1$. Moreover, for $1 \leq k<r, W_{k}$ is the set of new permutations added in the expansion of $e_{\left(0^{k}, i_{1}, i_{2}, \ldots, i_{n}\right)}$ from $e_{\left(0^{k-1}, i_{1}, i_{2}, \ldots, i_{n}\right)}$.

To prove Proposition 4.4, we need a lemma, which uses the Pieri rule.

Proposition 4.5 (Pieri rule) Define the following operator on Schubert polynomials:

$$
T_{i, j} \mathfrak{S}_{w}= \begin{cases}\mathfrak{S}_{w t_{i, j}} & \text { if } \ell\left(w t_{i, j}\right)=\ell(w)+1 \\ 0 & \text { otherwise. }\end{cases}
$$

We have

$$
e_{r}^{k} \mathfrak{S}_{w}=\sum T_{i_{1}, j_{1}} T_{i_{2}, j_{2}} \cdots T_{i_{r}, j_{r}} \mathfrak{S}_{w},
$$

summing over $i_{1}, \ldots, i_{r} \leq k<j_{1}, \ldots, j_{r}$, such that $i_{1}, \ldots, i_{r}$ are all distinct and $j_{1} \leq$ $\cdots \leq j_{r}$.

Lemma 4.6 The unique expansion of $e_{i}^{j} \mathfrak{S}_{w}=\sum_{w \in W} \beta_{w}^{I} \mathfrak{S}_{w}$ into Schubert polynomials has strong stable property, i.e., there exist $r$ such that for all $k \geq r$, we have

$$
e_{i}^{j+k} \mathfrak{S}_{1^{k} \times w}=\sum_{w \in W} \beta_{w}^{I} \mathfrak{S}_{1^{k} \times w}+\sum_{w_{1} \in W_{1}} \beta_{w_{1}}^{I} \mathfrak{S}_{1^{k-1} \times w}+\cdots+\sum_{w_{k} \in W_{r}} \beta_{w_{r}}^{I} \mathfrak{S}_{1^{k-r} \times w},
$$

where for $i=1, \ldots, r$, we have $W_{i} \neq \emptyset$. Moreover, for $1 \leq k<r, W_{k}$ is the set of new permutations added to the expansion of $e_{i}^{j+k} \mathfrak{S}_{1^{k} \times w}$ compared to the expansion of $e_{i}^{j+k-1} \mathfrak{S}_{1^{k-1} \times w}$.

Proof Use Proposition 4.5. Let $m$ be the minimal number of simple transformations we need in order to move the letter 1 to a position after $j$ in $w$. In the next step, we consider $e_{i}^{j+1} \mathfrak{S}_{1 \times w}$. We will get new terms if we can exchange the letter 1 in $1 \times w$ 
with some other letter. Then we can show that $e_{i}^{j+k} \mathfrak{S}_{1^{k} \times w}$ will have new terms in the expansion for all $0<k \leq i-m$ and there will be no new terms if $k>i-m$. In step $k=i-m$, there is exactly one new permutation.

Proof of Proposition 4.4 Use Lemma 4.6 from left to right, with $e_{i_{1}}^{1}=\mathfrak{S}_{w}$,

$$
\left(\left(e_{i_{1}}^{1} e_{i_{2}}^{2}\right) e_{i_{3}}^{3}\right) \cdots e_{i_{n}}^{n}
$$

For $e_{i_{1}}^{1} e_{i_{2}}^{2}$, let $\mathfrak{S}_{w_{1}}$ be the last new term added at step $n_{1}=i_{2}-1$. Then consider $\mathfrak{S}_{w_{1}} e_{i_{3}}^{k+n_{1}}$, let $\mathfrak{S}_{w_{2}}$ be the last new term at step $n_{1}+n_{2}$, with $n_{2}=i_{3}-1$, etc. We have the last new term added at step $N=n_{1}+\cdots+n_{n}-n$, is $\mathfrak{S}_{23} \cdots(|I|+1) 1$. Before this step, there are always new terms being added to the expansion.

For the expansion of $\mathfrak{S}_{w}$ into $e_{I}$, we have the following stable property:

Proposition 4.7 For the unique expansion $\mathfrak{S}_{w}=\sum_{I \in N^{\infty}} a_{I}^{w} e_{I}$, we have weak stable property, i.e., in the expansion $\mathfrak{S}_{1 \times w}=\sum_{J \in N^{\infty}} b_{J}^{1 \times w} e_{J}$, we have

$$
b_{0 I}^{1 \times w}=a_{I}^{w} .
$$

To prove this stable property, we use the following two lemmas:

Lemma 4.8 ([19, p. 31]) For $w=w_{1} \cdots w_{n} \in S_{n}$, we have

$$
\mathfrak{S}_{w w_{0}}=\sum_{a} K_{a, w}^{-1} e_{w_{0}\left(\rho_{n}-a\right)}
$$

where $w_{0}=n(n-1) \cdots 1, \rho_{n}=(n-1, n-2, \ldots, 1,0)$, and $K^{-1}=\left(K_{a, w}^{-1}\right)$ is the inverse of $K$.

Lemma 4.9 ([19, Proposition 17.3]) For any $u \in S_{n}$ and $a \in \mathbf{N}^{\infty}$, we have

$$
K_{a, u}^{-1}=\sum_{w \in S_{n}}(-1)^{\ell(w)} K_{w_{0} u, w\left(\rho_{n}\right)-a},
$$

where $w$ acts on a vector as rearranging the coordinates, e.g., $312(2,1,0)=(0,2,1)$.

Proof of Proposition 4.7 Consider Lemma 4.8. Notice that $w_{0}\left(\rho_{n}-a\right)=\left(0-a_{n}, 1-\right.$ $\left.a_{n-1}, \ldots, n-1-a_{1}\right)$, and $w_{0}\left(\rho_{n+1}-b\right)=\left(0-b_{n+1}, 1-b_{n}, \ldots, n-b_{1}\right)$. Assume $w_{0}\left(\rho_{n+1}-b\right)=\left(0, w_{0}\left(\rho_{n}-a\right)\right)$. Then $b=(a+1,0)$. Assume $1 \times w w_{0}=u w_{0}$. Then $u=(n+1) \times w$. So to prove the result, it suffices to show that

$$
K_{(a+1,0),(1+n) \times u}^{-1}=K_{a, u}^{-1} .
$$

Then by Lemma 4.9, we have

$$
K_{(a+1,0),(1+n) \times u}^{-1}=\sum_{w \in S_{n+1}}(-1)^{\ell(w)} K_{w_{0}((1+n) \times u), w\left(\rho_{n+1}\right)-(a+1,0)} .
$$


Notice that $w_{0}((1+n) \times u)=u$ and $w\left(\rho_{n+1}\right)-(a+1,0)=w\left(\rho_{n}\right)-a$ if $w\left(\rho_{n+1}\right)_{n+1}=0$. But if $w\left(\rho_{n+1}\right)_{n+1} \neq 0, w\left(\rho_{n+1}\right)-(a+1,0) \notin \mathbf{N}^{\infty}$, so $K_{w_{0}((1+n) \times u), w\left(\rho_{n+1}\right)-(a+1,0)}=0$. Therefore, $K_{(a+1,0),(1+n) \times u}^{-1}=K_{a, u}^{-1}$.

Remark 4.10 Consider the expansion of two Schubert polynomials $\mathfrak{S}_{w} \mathfrak{S}_{u}$ into Schubert polynomials again as we studied in Theorem 1.3. By Proposition 4.7, we can get a stabilized expansion of $\mathfrak{S}_{w}$ into the $e_{I}$ 's. Then, by Lemma 4.6, the expansion of each term $e_{I} \mathfrak{S}_{u}$ into Schubert polynomials stabilizes. This way, we get a second proof of Theorem 1.3.

Acknowledgements I thank Richard Stanley, Alex Postnikov and Steven Sam for helpful discussions. I am also very grateful for the anonymous referees for many very good suggestions.

\section{References}

1. Assaf, S., McNamara, P.: A Pieri rule for skew shapes. J. Comb. Theory, Ser. A 118, 277-290 (2011)

2. Assaf, S., Bergeron, N., Sottile, F.: On the multiplication of Schubert polynomials. In preparation

3. Bergeron, N., Billey, S.: RC-graphs and Schubert polynomials. Exp. Math. 2, 257-269 (1993)

4. Bernstein, I.N., Gelfand, I.M., Gelfand, S.I.: Schubert cells and cohomology of the spaces G/P. Russ. Math. Surv. 28, 1-26 (1973)

5. Billey, S., Jockusch, W., Stanley, R.P.: Some combinatorial properties of Schubert polynomials. J. Algebr. Comb. 2, 345-374 (1993)

6. Demazure, M.: Désingularisation des variétés de Schubert généralisées. Ann. Sci. Ec. Norm. Super. (4) 7, 53-88 (1974)

7. Fomin, S., Greene, E., Reiner, V., Shimozono, M.: Balanced labellings and Schubert polynomials. Eur. J. Comb. 18, 373-389 (1997)

8. Gelfand, I., Krob, D., Lascoux, A., Leclerc, B., Retakh, V.S., Thibon, J.-Y.: Noncommutative symmetric functions. Adv. Math. 112(2), 218-348 (1995)

9. Knutson, A., Yong, A.: A formula for K-theory truncation Schubert calculus. Int. Math. Res. Not. 70, 3741-3756 (2004)

10. Kohnert, A.: Multiplication of a Schubert polynomial by a Schur polynomial. Ann. Comb. 1, 367-375 (1997)

11. Lam, T., Lauve, A., Sottile, F.: Skew Littlewood-Richardson rules for Hopf algebras. IMRN (2010). arXiv:0908.3714

12. Lascoux, A., Schützenberger, M.P.: Polynômes de Schubert. C. R. Acad. Sci. Paris 294, 447-450 (1982)

13. Lascoux, A., Schützenberger, M.P.: Schubert polynomials and the Littlewood-Richardson rule. Lett. Math. Phys. 10, 111-124 (1985)

14. Lascoux, A., Schützenberger, M.P.: Fonctorialité de polynômes de Schubert. Contemp. Math. 88, 585-598 (1989)

15. Lenart, C.: Growth diagrams for the Schubert multiplication. J. Comb. Theory, Ser. A 117, 842-856 (2010)

16. Macdonald, I.G.: Notes on Schubert polynomials. Laboratorie de Combinatoire et d'Informatique Mathématique, Université du Québec à Montréal (1991)

17. Manivel, L.: Symmetric Functions, Schubert Polynomials and Degeneracy Loci. Am. Math. Soc., Providence (2001)

18. Monk, D.: Geometry of flag manifolds. Proc. Lond. Math. Soc. 9, 253-286 (1959)

19. Postnikov, A., Stanley, R.: Chains in the Bruhat order. J. Algebr. Comb. 46(29), 133-174 (2009)

20. Sottile, F.: Pieri's rule for flag manifolds and Schubert polynomials. Ann. Inst. Fourier 46, 89-110 (1996)

21. Stanley, R.P.: On the number of reduced decompositions of elements of Coxeter groups. Eur. J. Comb. 5, 359-372 (1984)

22. Winkel, R.: On the multiplication of Schubert polynomials. Adv. Appl. Math. 20, $73-97$ (1998) 